\title{
Orbital polarization of $\mathrm{LaSrMnO}_{4}$ studied by soft X-ray linear dichroism
}

\author{
W.B. Wu ${ }^{a, b}$, D.J. Huang ${ }^{a, b, *}$, G.Y. Guo ${ }^{\text {b,c }}$, H.-J. Lin ${ }^{b}$, T.Y. Hou ${ }^{\text {b }}$, C.F. Chang ${ }^{\text {, }}$,

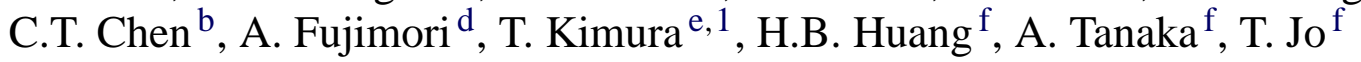 \\ ${ }^{a}$ Department of Electrophysics, National Chiao-Tung University, Hsinchu 300, Taiwan, ROC \\ ${ }^{\mathrm{b}}$ National Synchrotron Radiation Research Center, Hsinchu 300, Taiwan, ROC \\ ${ }^{\mathrm{c}}$ Department of Physics, National Taiwan University, Taipei 106, Taiwan, ROC \\ ${ }^{\mathrm{d}}$ Department of Complexity Science and Department of Physics, University of Tokyo, Tokyo 113-0033, Japan \\ ${ }^{\mathrm{e}}$ Department of Applied Physics, University of Tokyo, Tokyo 113-8656, Japan \\ ${ }^{\mathrm{f}}$ Department of Quantum Matters, ADSM, Hiroshima University, Higashi-Hiroshima 739-8530, Japan
}

Available online 1 April 2004

\begin{abstract}
With polarization-dependent soft X-ray absorption, we determined the orbital character of occupied and the lowest unoccupied $e_{g}$ orbitals of prototype single-layered perovskite $\mathrm{LaSrMnO}_{4}$. Our measurements indicate that $\mathrm{LaSrMnO}_{4}$ exhibits strong orbital polarization in the $e_{g}$ states of $\mathrm{Mn}^{3+}$ sites, leading us to conclude that $\mathrm{LaSrMnO}_{4}$ has a "ferro-orbital" ordering of $3 z^{2}-r^{2}$. We found that linear dichroism in soft $\mathrm{X}$-ray absorption is an effective experimental method to identify the nature of orbital ordering in transition-metal oxides such as manganites. (C) 2004 Elsevier B.V. All rights reserved.
\end{abstract}

Keywords: Polarization; Prototype; Perovskite

\section{Introduction}

Orbital ordering is one of the important subjects in current research of transition metal compounds [1]. In some compounds, there is a real space ordering of the charge carrier in certain orbitals, i.e., orbital ordering, resulting from superexchange interaction, lattice distortion, and Coulomb correlation of electrons. Orbital ordering manifests itself in the site dependent orientation of the quadrupole moment as a result of the spatial distribution of the outermost valence $d$ electrons. Orbital ordering gives rise to the anisotropy of the electron-transfer interaction. As the magnetic and transport properties are closely correlated to the orbital and charge degrees of freedom, there have been many efforts to clarify the microscopic mechanisms giving rise to such exotic phenomena. For example, a fundamental issue in the physics of the manganites concerns the mechanism of the charge-orbital ordering and charge stripe formation. The mechanism of charge-orbital ordering is being extensively discussed [2-9].

\footnotetext{
* Corresponding author.

E-mail address: djhuang@nsrrc.org.tw (D.J. Huang).

${ }^{1}$ Present address: Los Alamos National Laboratory, MST-MISL, Mail Stop K774, Los, Alamos, NM 87545, USA
}

Measurements of resonant X-ray scattering (RXS) at the $\mathrm{Mn} 1 \mathrm{~s}$ the threshold have been argued for the evidence of $3 \mathrm{~d}$ orbital ordering of manganites [10,11]. In one of the interpretations of RXS measurements, a Mn 1s electron is first excited to the unoccupied $\mathrm{Mn} 4 \mathrm{p}$ state by absorbing the incident photon and then a photon is emitted resulting from the $4 p \rightarrow 1$ s de-excitation. Consequently, the ordering of $4 p$ orbitals, e.g., the alternately arrangement of $4 p_{x}$ and $4 p_{y}$ orbitals of $\mathrm{Mn}$ in $\mathrm{LaMnO}_{3}$, is directly observed. Through intra-atomic 3d-4p multipole interaction, ordering of Mn $3 \mathrm{~d}$ orbitals is indirectly observed. However, such an interpretation of RXS at Mn K-edge is controversial. Orbital ordering in transition-metal oxides is typically accompanied by Jahn-Teller lattice distortion. Calculations based on a local-density approximation including on-site Coulomb interactions $(\mathrm{LDA}+\mathrm{U})[12,13]$ and multiple scattering theory [14] indicate that RXS measurements pertain mainly to Jahn-Teller distortion, instead of directly observing $3 \mathrm{~d}$ orbital ordering. To identify the orbital character of $3 \mathrm{~d}$ states in orbital-ordered manganites is an important subject.

Polarization-dependent soft X-ray absorption (XAS), particularly linear dichroism, provides us with a powerful means to identify spin and orbital occupation of transition-metal oxides. For example, linear dichroism (LD) in XAS measurements on $\mathrm{V}_{2} \mathrm{O}_{3}$ enabled important 
observations on its electronic structure, identifying the orbital occupation of $\mathrm{V} 3 \mathrm{~d}^{2}$ ions in various phases, defining that they are in a high-spin state, i.e., $S=1$ [15].

Calculations based on a single-ion model which includes the $3 \mathrm{~d}-3 \mathrm{~d}$ and $3 \mathrm{~d}-2 \mathrm{p}$ multipole interactions and a crystal field have recently shown that the linear dichroism in $\mathrm{Mn} \mathrm{L}_{2,3}$ absorption exhibits multiplet structure and strongly reflect $3 \mathrm{~d}$ orbital polarization, but insensitive to the Jahn-Teller distortion of the crystal $[16,17]$. Linear dichroism therefore is expected to be a promising method to identify the orbital character of $3 \mathrm{~d}$ electrons of orbital ordered compounds.

$\mathrm{LaSrMnO}_{4}$, a mother compound of single-layered manganites $\mathrm{La}_{1-x} \mathrm{Sr}_{1+x} \mathrm{MnO}_{4}$, has the $\mathrm{K}_{2} \mathrm{NiF}_{4}$ structure and is an antiferromagnetic insulator. In the single-layered perovskite structure of $\mathrm{LaSrMnO}_{4}$, there exhibits a strong Jahn-Teller lattice distortion in which the $\mathrm{Mn}-\mathrm{O}$ bond length along the $z$-axis is significantly larger than that in the $a b$ plane, i.e., the $\mathrm{MnO}_{2}$ plane. Consequently, the $e_{g}$ band of $\mathrm{Mn}$ is preferentially occupied by the $\mathrm{d}_{3 z^{2}-r^{2}}$ orbital as a result of the bigger bond length of $\mathrm{Mn}-\mathrm{O}$ along the $z$-axis; the lowest unoccupied $e_{g}$ orbital is $\mathrm{d}_{x^{2}-y^{2}}$. Figure 1 displays the $\mathrm{Mn} 3 \mathrm{~d}$ partial density of states of $\mathrm{LaSrMnO}_{4}$ in the vicinity of the Fermi level obtained from band structure calculations in the scheme LDA $+\mathrm{U}$ [18], showing that $\mathrm{LaSrMnO}_{4}$ exhibits strong orbital polarization in the $e_{g}$ band. Because of its large Jahn-Teller splitting, $\mathrm{LaSrMnO}_{4}$ is a good system for examining whether LD measurements can provide us with information on the orbital character of $3 \mathrm{~d}$ electrons.

In this paper, we present measurements and multiplet calculations of linear dichroism in Mn 2p-edge XAS to identify the $3 \mathrm{~d}$ orbital character of the $e_{g}$ electrons in $\mathrm{LaSrMnO}_{4}$. Our results clearly demonstrate that linear dichroism in soft $\mathrm{X}$-ray absorption is a powerful experiment tool in determining the nature of orbital ordering in $3 \mathrm{~d}$ transition metal oxides.

The rest of this paper is organized as follows. In the next section, we briefly describe the experimental details

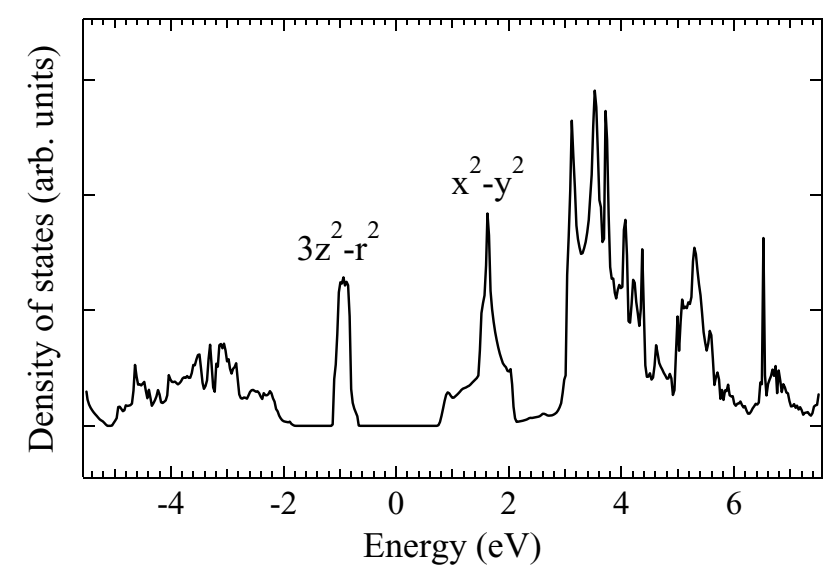

Fig. 1. Partial density of states of $\mathrm{Mn} 3 \mathrm{~d}$ in $\mathrm{LaSrMnO}_{4}$ obtained from $\mathrm{LDA}+\mathrm{U}$ calculations with $U=8 \mathrm{eV}$. The Fermi level is the zero of energy.

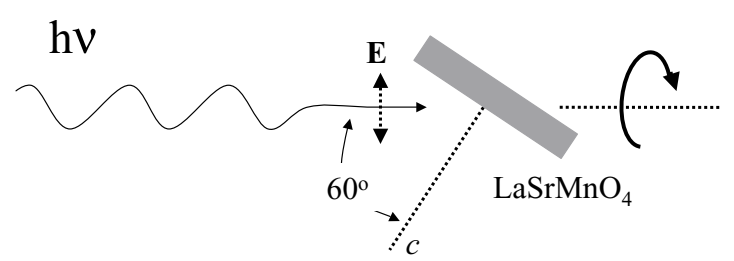

Fig. 2. Schematic illustration of sample rotation for LD measurements. The sample was rotated about the direction of incident photons to obtain two sets of XAS spectra with $\boldsymbol{E} \perp c$ and with $\boldsymbol{E}$ in the plane defined by the $c$-axis and the direction of incident radiation. Polarization-dependent XAS was deduced as described in the text.

including growth and characterizations of $\mathrm{LaSrMnO}_{4}$ single crystal, and measurements of linear dichroism. Methods of multiplet are briefly summarized in Section 3. Results and discussion are presented in Section 4, followed by conclusions.

\section{Experimental}

Single-crystalline samples of $\mathrm{LaSrMnO}_{4}$ were grown by the floating zone method, as described elsewhere [19]. Measurements of X-ray diffraction at room temperature show that our samples are of single phase and have the $I 4 / \mathrm{mmm}$ tetragonal structure with lattice constants $c=13.04 \AA$ and $a=3.81 \AA$ A. The crystal also exhibits large Jahn-Teller distortion, i.e., the $\mathrm{Mn}-\mathrm{O}$ bond length of $2.29 \AA$ along the $c$-axis and $1.90 \AA$ along the $a$-axis and $b$-axis.

XAS measurements on $\mathrm{LaSrMnO}_{4}$ single crystals at various temperatures were performed at the Dragon beamline of the National Synchrotron Radiation Research Center in Taiwan. We recorded XAS spectra by collecting the sample drain current at $300 \mathrm{~K}$. Crystals were in situ cleaved in an ultra-high vacuum at $90 \mathrm{~K}$; the incident angle was $60^{\circ}$ from the sample surface normal and the photon energy resolution was $0.2 \mathrm{eV}$. As shown in Fig. 2, we rotated the sample about the direction of incident photons to obtain LD spectra from which experimental artifacts related to the difference in the optical path and to the probing area have been eliminated. All measured XAS spectra referred to the $\boldsymbol{E}$ vector parallel to the $c$-axis are shown with a correction for the geometry effect. A geometry effect in XAS measurements is corrected as follows: $I_{\|}=4 / 3\left(I-\left(1 / 4 I_{\perp}\right)\right)$, in which $I_{\perp}$ and $I$ are measured XAS intensities with $\boldsymbol{E} \perp c$ and with $\boldsymbol{E}$ in the plane defined by the $c$-axis and the direction of incident radiation, respectively; $I_{\|}$is the deduced XAS intensity for $\boldsymbol{E} \| c$.

\section{Multiplet calculations}

We used a cluster model based on a configuration interaction (CI) approach to calculate XAS and linear dichroism spectra of $\mathrm{LaSrMnO}_{4}[20,21]$. In the calculation, octahedral 
$\mathrm{MnO}_{4}^{5-}$ clusters were used. Because of strong $\mathrm{Mn} 3 \mathrm{~d}-\mathrm{O} 2 \mathrm{p}$ hybridization, the electronic state of the $\mathrm{Mn}^{3+}$ ions is described as a linear combination of the $3 \mathrm{~d}^{4}, 3 \mathrm{~d}^{5} \underline{\mathrm{L}}, 3 \mathrm{~d}^{6} \underline{\mathrm{L}}^{2}$, where $\underline{L}$ denotes a hole on oxygen $2 p$ orbital. We calculated the spectra by exactly diagonalizing the single-ion Hamiltonian under the crystal field where multipole interactions between the $3 \mathrm{~d}-3 \mathrm{~d}$ and $3 \mathrm{~d}-2 \mathrm{p}$ core are described by the Slater integrals and the spin-orbit interactions. The "non multiplet" Hamiltonian describing valence and core states and the effective interaction between electrons are expressed in terms of the $3 \mathrm{~d}-3 \mathrm{~d}$ and $3 \mathrm{~d}-2 \mathrm{p}$ Coulomb interactions $\left(U_{\mathrm{dd}}\right.$ and $U_{\mathrm{dc}}$, respectively), and the hybridization strength between $\mathrm{Mn}$ $3 \mathrm{~d}$ and $\mathrm{O} 2 \mathrm{p}$. In addition, the charge fluctuation between the ligand $2 \mathrm{p}$ and cation $3 \mathrm{~d}$ is also taken into account with charge-transfer energy $\Delta$ defined as $\left(E_{3 \mathrm{~d}^{5} \mathrm{~L}}-E_{3 \mathrm{~d}^{4}}\right)$, where $E_{3 \mathrm{~d}^{5} \mathrm{~L}}$ and $E_{3 \mathrm{~d}^{4}}$ are the average energies of $3 \mathrm{~d}^{5} \underline{\mathrm{L}}$ and $3 \mathrm{~d}^{4}$ configurations, respectively.

The Hatree-Fock values were adopted for the coupling constants of the $2 \mathrm{p}$-core and $3 \mathrm{~d}$ spin-orbit interactions and $80 \%$ of the values were used for the Slater integrals, which describe the $3 d-3 d$ and $3 d-2 p$ multiplet interactions in the Hamltonia $[22,23]$. The values of charge-transfer energy $\Delta$, on-site Coulomb energies $U_{\mathrm{dd}}$ and $U_{\mathrm{dc}}$, and hopping integrals $\left(V\left(t_{2 g}\right)\right.$ and $\left.V\left(e_{g}\right)\right)$ between $3 \mathrm{~d}$ and oxygen $2 \mathrm{p}$ molecular orbitals were included to reproduce the experimental spectra. In addition, we also used octahedral crystal field parameter $10 \mathrm{Dq}=\varepsilon_{\mathrm{d}}\left(e_{g}\right)-\varepsilon_{\mathrm{d}}\left(t_{2 g}\right)$, and hybridization strength between the $\mathrm{O} 2 \mathrm{p}$ orbitals $2 T_{\mathrm{pp}}=\varepsilon_{\mathrm{L}}\left(e_{g}\right)-\varepsilon_{\mathrm{L}}\left(t_{2 g}\right)$ as the calculation parameters. To reduce the number of the free parameters, the empirical relation between Slater-Koster hopping integrals $p d \sigma \approx-4 / \sqrt{3} p d \pi$ was assumed.

\section{Results and discussion}

To experimentally confirm the strong $3 \mathrm{~d}$ orbital polarization of $\mathrm{LaSrMnO}_{4}$ concluded from LDA + U band structure calculations, we performed O 1s XAS. Absorption at the oxygen K-edge is a dipole allowed transition in which an $\mathrm{O} 1 \mathrm{~s}$ electron is excited to unoccupied $\mathrm{O} 2 \mathrm{p}$ orbitals. The pre-edge structure, i.e., the structure at the absorption threshold, is determined by the hybridization between $\mathrm{O} 2 \mathrm{p}$ and $\mathrm{Mn} \mathrm{3d}$ bands. In addition, at slightly higher energies, the XAS structure is determined unoccupied orbitals corresponding to the hybridization between the oxygen and the $\mathrm{La} 4 \mathrm{f} / 5 \mathrm{~d}$ and $\mathrm{Sr} 3 \mathrm{~d}$ orbitals. In configuration approach, the strength of $\mathrm{O} 1 \mathrm{~s} \rightarrow 2 \mathrm{p}$ transition of $\mathrm{LaSrMnO}_{4}$ is determined by the weight of $3 \mathrm{~d}^{5} \underline{\mathrm{L}}$ character in the ground state. Polarization-dependent O 1s XAS therefore reflects the symmetry of unoccupied Mn 3d orbitals. Fig. 3 depicts O 1s XAS spectra of $\mathrm{LaSrMnO}_{4}$ with the $\boldsymbol{E}$ vector of photons perpendicular and parallel to the crystal $c$-axis. Our measurements reveal that the lowest unoccupied $\mathrm{O} 2 \mathrm{p}$ bands have a strong in-plane character, consistent with the conclusion obtained from LDA + U calculations, indicating that $\mathrm{LaSrMnO}_{4}$ exhibits $x^{2}-y^{2}$ orbital character in its unoccupied $e_{g}$ band. It

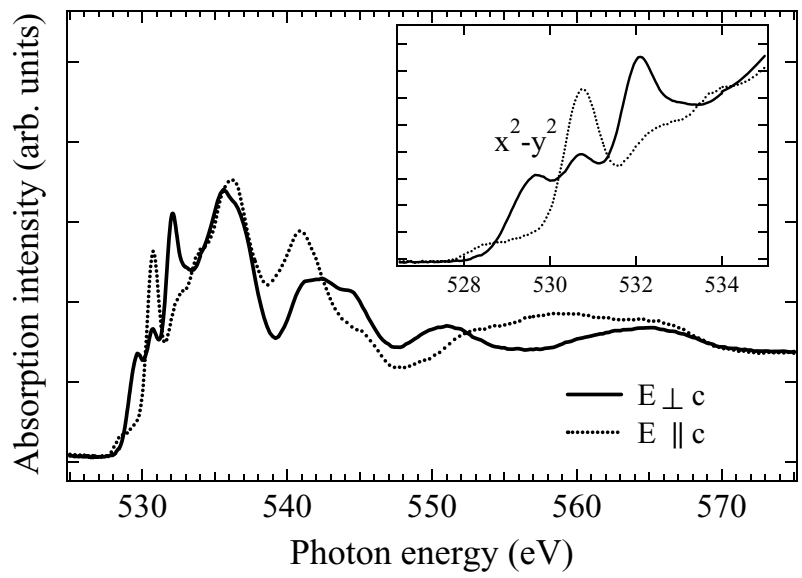

Fig. 3. Polarization-dependent XAS of $\mathrm{LaSrMnO}_{4}$ single crystal. The solid and dashed lines show the spectra taken with the $\boldsymbol{E}$ vector of photons perpendicular and parallel to the crystal $c$-axis.

is interesting to note that the pre-edge results of our $\mathrm{O} 1 \mathrm{~s}$ measurements are nearly identical to those of previous measurements with in situ cleaved samples [24], but in contrast to those of specially cut samples with annealing under $\mathrm{Ar}$ atmosphere [25].

After establishing the $3 z^{2}-r^{2}$ orbital character in the occupied $e_{g}$ band of $\mathrm{LaSrMnO}_{4}$, we now present LD measurements in the Mn L-edge absorption. Fig. 4 shows the polarization-dependent XAS spectra of $\mathrm{LaSrMnO}_{4}$ taken with the $\boldsymbol{E}$ vector of photons perpendicular $(\boldsymbol{E} \perp c)$ and parallel $(\boldsymbol{E} \| c)$ to the crystal $c$-axis. Since the unoccupied $e_{g}$ band has a strong orbital anisotropy, the cross section of $\mathrm{Mn}$ $2 \mathrm{p} \rightarrow 3 \mathrm{~d}$ absorption excited by photons with in-plane polarization $(\boldsymbol{E} \perp c)$ is larger than that with out-of-plane polarization $(\boldsymbol{E} \| c)$, if the multiplet effect resulting from $3 \mathrm{~d}-3 \mathrm{~d}$ and $2 \mathrm{p}-3 \mathrm{~d}$ multipole interactions is neglected. In $\mathrm{L}_{3}$ region,

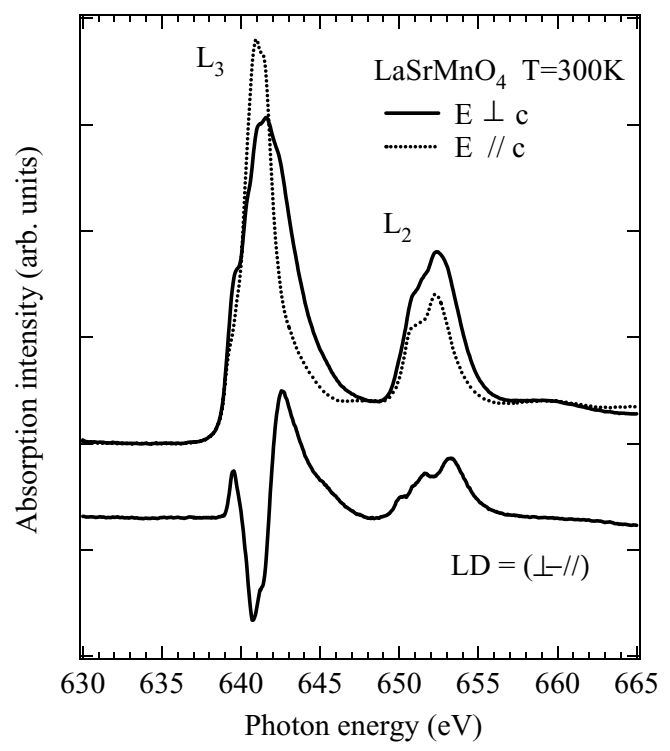

Fig. 4. Measured LD and polarization-dependent XAS taken with $\boldsymbol{E} 4 \perp c$ (solid line) and $\boldsymbol{E} \| c$ (dashed line) of $\mathrm{LaSrMnO}_{4}$. 


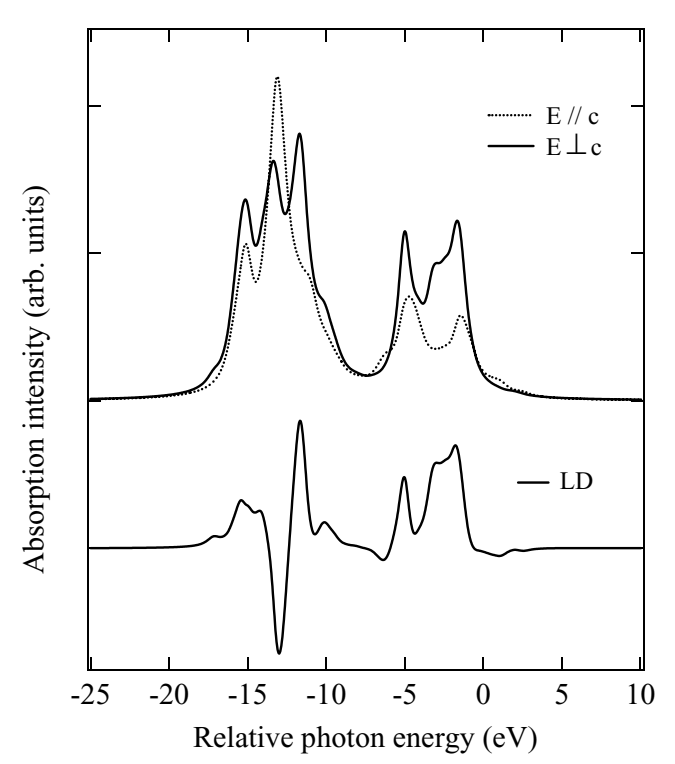

Fig. 5. Calculated LD and polarization-dependent XAS of $\mathrm{Mn}^{3+}$ with $3 d_{3^{2}-r^{2}}$ orbital occupied. The descriptions for the figure correspond to those of Fig. 4.

the four core $2 p$ states contribute to $3 d-2 p$ multipole interaction and multiplet structures, while the two states in $\mathrm{L}_{2}$ region. The difference in XAS between the two linear polarizations is therefore remarkable in $\mathrm{L}_{3}$ region compared with $\mathrm{L}_{2}$ region. By an inspection of $2 \mathrm{p}-3 \mathrm{~d}$ multipole interaction described by the Gaunt coefficient, the XAS spectral width for $\boldsymbol{E}$ perpendicular to $c$ is found to be larger than that for $\boldsymbol{E}$ parallel to $c$ both in $\mathrm{L}_{3}$ and $\mathrm{L}_{2}$, if $3 z^{2}-r^{2}$ is occupied. This causes the basic LD feature especially in $\mathrm{L}_{3}$, although less prominent in $\mathrm{L}_{2}$. Our measurements of linear dichroism indeed show that the integrated intensity of XAS taken with in-plane polarization is larger than that with out-of-plane polarization, consistent with the above argument.

To further understand the LD spectrum of $\mathrm{LaSrMnO}_{4}$, we performed multiplet calculations to obtain polarization dependent XAS. In our calculations, we assumed $10 \mathrm{Dq}=$ $2.2 \mathrm{eV}$. Other parameters are the same as those used in Ref. [16]. We used parameters $\Delta_{i}$ and $\Delta_{f}$ to represent the energy difference between $3 \mathrm{~d}_{3^{2}-r^{2}}$ and $3 \mathrm{~d}_{x^{2}-y^{2}}$ orbitals in the initial and the final states of XAS, respectively; we assumed that $3 \mathrm{~d}_{3^{2}-r^{2}}$ has lower energy than $3 \mathrm{~d}_{x^{2}-y^{2}}$ for $\Delta_{i}$ and $\Delta_{f}$ positive. $\Delta_{i}$ and $\Delta_{f}$ result from the contribution of the molecular field and the Jahn-Teller distortion; the former is $\sim 0.1 \mathrm{eV}$ at temperature below the transition, while the latter can be several tenth of an $\mathrm{eV}$. By comparing the LD spectra of different values of $\Delta_{i}$ and $\Delta_{f}$, we found that the LD spectrum is weakly dependent on $\Delta_{f}$ and the basic feature of LD spectrum is determined by $3 \mathrm{~d}$ electron distribution in the initial state. In other words, LD is insensitive to the Than-Teller distortion of the $\mathrm{MnO}_{6}$ cluster [16]. With $\Delta_{i}=0.1 \mathrm{eV}$, and $\Delta_{f}=0.5 \mathrm{eV}$, Fig. 5 shows calculated LD and polarization-dependent XAS of $\mathrm{Mn}^{3+}$ with $3 \mathrm{~d}_{3^{2}-r^{2}}$ orbital occupied; the spectra taken with $\boldsymbol{E} \perp c$ and $\boldsymbol{E} \| c$ are plotted by solid and dashed lines, respectively.
Most of the features in the measured LD at Mn L-edge are reproduced by multiplet calculations for $\mathrm{Mn}^{3+}$ ions with occupied $\mathrm{d}_{3 z^{2}-r^{2}}$ orbitals, revealing that LD in L-edge XAS is an effective means to examine the orbital character of $3 d$ electronic states in an orbital-ordered compound.

The comparison of measurements of polarizationdependent XAS with multiplet calculations leads us to suggest that one can use LD in L-edge X-ray absorption to identify the nature of orbital ordering in transition metal compounds such as the orbital nature of half-doped single-layered manganites which exhibit CE-type antiferromagnetic ordering and charge-orbital ordering $[10,19,26,27]$. Below a charge ordering temperature $T_{\mathrm{CO}}=$ $217 \mathrm{~K}$, the valence of $\mathrm{La}_{0.5} \mathrm{Sr}_{1.5} \mathrm{MnO}_{4}$ orders in an alternating pattern with two distinct sites identified as $\mathrm{Mn}^{3+}$ and $\mathrm{Mn}^{4+}[19,27]$. Electron-lattice coupling and Coulomb correlations give rise to alternating orbital ordering. In contrast to the $3 z^{2}-r^{2}$ "ferro-orbital" ordering in $\mathrm{LaSrMnO}_{4}, e_{g}$ electrons of the $\mathrm{Mn}^{3+}$ sites in $\mathrm{La}_{0.5} \mathrm{Sr}_{1.5} \mathrm{MnO}_{4}$ are believed to exhibit an orbital ordering of $3 x^{2}-r^{2} / 3 y^{2}-r^{2}$, in which occupied $\mathrm{d}_{3 x^{2}-r^{2}}$ and $\mathrm{d}_{3 y^{2}-r^{2}}$ orbitals are alternately arranged at two sublattices in the $a b$ plane. However, $\mathrm{d}_{3 x^{2}-r^{2}}$ and $\mathrm{d}_{x^{2}-z^{2}}\left(\mathrm{~d}_{3 y^{2}-r^{2}}\right.$ and $\left.\mathrm{d}_{y^{2}-z^{2}}\right)$ orbitals might be mixed, because orbitals of these two types have the same spatial symmetry in the $\mathrm{MnO}_{2}$ plane. One thus can apply the technique of LD in soft X-ray absorption to clarify the orbital character of $3 \mathrm{~d}$ electrons in the $\mathrm{Mn} e_{g}$ band of $\mathrm{La}_{0.5} \mathrm{Sr}_{1.5} \mathrm{MnO}_{4}$. Furthermore, in principle, one could directly observe both orbital ordering and Jahn-Teller ordering in manganites by using $\mathrm{LD}$ in resonant $\mathrm{X}$-ray scattering at $\mathrm{Mn}_{2,3}$-edges.

\section{Conclusions}

We have studied the orbital polarization of $3 \mathrm{~d}$ electrons in the $\mathrm{Mn} e_{g}$ band of $\mathrm{LaSrMnO}_{4}$. The results demonstrate that LD in Mn 2p XAS is a powerful method to test the validity of models for orbital ordering in transition-metal oxides. This technique would open up a new avenue for determining the orbital character of $3 \mathrm{~d}$ electrons in transition metal oxides, and possibly examining the mechanism of orbital ordering.

\section{Acknowledgements}

We thank L.H. Tjeng for loaning his XAS chamber. This work was supported in part by the National Science Council of Taiwan and by a Grant-in-Aid for Scientific Research in Priority Area "Novel Quantum Phenomena in Transition-Metal Oxides" from the Ministry of Education, Culture, Sports, Science and Technology of Japan.

\section{References}

[1] Y. Tokura, N. Nagaosa, Science 288 (2000) 462.

[2] T. Mizokawa, A. Fujimori, Phys. Rev. B 56 (1997) 493.

[3] T. Mutou, H. Kontani, Phys. Rev. Lett. 83 (1999) 3685. 
[4] D. Khomskii, J. van der Brink, Phys. Rev. Lett. 85 (2000) 3329.

[5] T. Hotta, E. Dagotto, H. Koizumi, Y. Takadal, Phys. Rev. Lett. 86 (2001) 2478 .

[6] T. Mutou, H. Kontani, Phys. Rev. Lett. 86 (2001) 2479.

[7] J. van der Brink, G. Khaliullin, Phys. Rev. Lett. 83 (1999) 5118.

[8] P. Mahadevan, K. Terakura, D.D. Sarma, Phys. Rev. Lett. 87 (2001) 66404.

[9] Z. Popovic, S. Satpathy, Phys. Rev. Lett. 88 (2002) 197201.

[10] Y. Murakami, H. Kawada, H. Kawata, M. Tanaka, T. Arima, Y. Moritomo, Y. Tokura, Phys. Rev. Lett. 80 (1998) 1932.

[11] Y. Murakami, J.P. Hill, D. Gibbs, M. Blume, I. Koyama, M. Tanaka, H. Kawata, T. Arima, Y. Tokura, K. Hirota, Y. Endoh, Phys. Rev. Lett. 81 (1998) 582.

[12] I.S. Elfimov, V.I. Anisimov, G.A. Sawatzky, Phys. Rev. Lett. 82 (1999) 4264.

[13] P. Benedetti, J. van den Brink, E. Pavarini, A. Vigliante, P. Wochner, Phys. Rev B 63 (2001) 60408.

[14] M. Benfatto, Y. Joly, C.R. Natoli, Phys. Rev. Lett. 83 (1999) 636.

[15] J.-H. Park, L.H. Tjeng, A. Tanaka, J.W. Allen, C.T. Chen, P. Metcalf, J.M. Honig, F.M.F. de Groot, G.A. Sawatzky, Phys. Rev. B 61 (2000) 11506.
[16] H.B. Huang, T. Shishidou, T. Jo, J. Phys. Soc. Jpn. 69 (2000) 2399.

[17] H.B. Huang, T. Jo, J. Phys. Soc. Jpn. 71 (2001) 3094.

[18] G.Y. Guo et al. (Unpublished).

[19] Y. Moritomo, Y. Tomioka, A. Asamitsu, Y. Tokura, Y. Matsui, Phys. Rev. B 51 (1995) 3297.

[20] A. Tanaka, T. Jo, J. Phys. Soc. Jpn. 63 (1994) 2788.

[21] A. Tanaka, T. Jo, J. Phys. Soc. Jpn. 61 (1992) 2669.

[22] F.M.F. de Groot, J.C. Fuggle, B.T. Thole, G.A. Sawatzky, Phys. Rev. B 42 (1990) 5459.

[23] T. Uozumi, K. Okada, A. Kotani, R. Zimmermann, P. Steiner, S. Hüfner, Y. Tezuka, S. Shi, J. Elect. Spect. Relat. Phenom. 83 (1997) 9.

[24] D. Dessau, Z.-X. Shen, in: Y. Tokura (Ed.), Colossal Magnetoresistive Oxides, Gordeorn and Breach Science Publishers, 2000, p. 149.

[25] J. van Elp, H. Sato, T. Kimura, T. Toda, Y. Okamura, Y. Tokura, M. Taniguchi, J. Phys. Soc. Jpn. 69 (2000) 2391.

[26] J.B. Goodenough, Phys. Rev. 100 (1955) 564.

[27] B.J. Sternlieb, J.P. Hill, U.C. Wildgruber, G.M. Luke, B. Nachumi, Y. Moritomo, Y. Tokura, Phys. Rev. Lett. 76 (1996) 2169. 